\title{
Phytochemical and biological features of Phyllanthus niruri and Phyllanthus urinaria harvested at different growth stages revealed by 1 H NMR-based metabolomics
}

\begin{abstract}
Several studies have suggested that plants are potential sources of bioactive compounds. Phyllanthus is a plant genus that has been used in traditional medicine due to its phytomedicinal metabolites content. The variation between two Phyllanthus species (P. niruri and P. urinaria) was studied using proton nuclear magnetic resonance (1H NMR) combined with multivariate data analysis (MVDA). The total phenolic content (TPC), DPPH radical scavenging activity and $\alpha$-glucosidase inhibitory activity of the Phyllanthus species were also evaluated and correlated with their phytochemical constituents at different growth stages ( 8,10 and 12 weeks) using partial least square regression (PLS). Principal component analysis (PCA) and PLS indicated separation between the two species based on the identified metabolites and the screened bioactivities. A comparison of the two species indicated that P. urinaria was separated from P. niruri due to its larger quantity of fatty and amino acids, choline, phyllanthin and sucrose. However, P. niruri contained higher quantities of hypophyllanthin and phenolic compounds. The loading column plot, which was used to compare the P. niruri at different growth stages, indicated that the eight-week-old plant contained a higher amount of fatty acids, amino acids (leucine and alanine), phyllanthin and choline. The dominant substances in the P. niruri at 10 weeks of growth by PC1 were identified as hypophyllanthin, malic acid, sucrose, and identified phenolics. The 12 week sample was differentiated by its higher sugars contents as well as malic acid and leucine. The harvested samples of both Phyllanthus species at ten weeks of age exhibited significant bioactivities with the highest content and number of metabolites.
\end{abstract}

Keyword: Phyllanthus species; Metabolomics; Growth stages; Proton nuclear magnetic resonance; Biological activities; UPLC-MS/MS 